\section{IMPACT OF A DISEASE SELF-MANAGEMENT PROGRAM ON EMPLOYEE HEALTH AND WORK PERFORMANCE: INTRODUCING WORKPLACE CDSMP}

M. Smith ${ }^{1}$, M. Wilson ${ }^{2}$, M. Robertson ${ }^{2}$, H. Padilla ${ }^{2}$, H. Zuercher' ${ }^{2}$ R. Vandenberg ${ }^{2}$, K. Lorig 3 , D. DeJoy², 1. Texas A\&M School of Public Health, 2. The University of Georgia, 3. Self-Management Resource Center

Disease management is gaining importance in workplace health promotion given the aging workforce and rising chronic disease prevalence. The Chronic Disease Self-Management Program (CDSMP) is an effective intervention widely offered in diverse community settings; however, adoption remains low in workplace settings. As part of a larger NIH-funded randomized-controlled trial, this study examines the effectiveness of a worksite-tailored version of CDSMP (wCDSMP [n=72]) relative to CDSMP ('Usual Care'[n=109]) to improve health and work performance among employees with one or more chronic conditions. Multiple-group latent-difference score models with sandwich estimators were fitted to identify changes from baseline to 6-month follow-up. Overall, participants were primarily female $(87 \%)$, non-Hispanic white $(62 \%)$, and obese $(73 \%)$. On average, participants were age 48 (range: $23-72$ ) and self-reported 3.25 chronic conditions (range: 1-16). The most commonly reported conditions were high cholesterol (45\%), high blood pressure $(45 \%)$, anxiety/emotional/mental health condition $(26 \%)$, and diabetes $(25 \%)$. Among wCDSMP participants, significant improvements were observed for physically unhealthy days $(\mathrm{u} \Delta=-2.07, \mathrm{P}=0.018)$, fatigue $(\mathrm{u} \Delta=-2.88, \mathrm{P}=0.002)$, sedentary behavior ( $\mathrm{u} \Delta=-4.49, \mathrm{P}=0.018)$, soda/sugar beverage consumption $(\mathrm{u} \Delta=-0.78, \mathrm{P}=0.028)$, and fast food intake ( $\mathrm{u} \Delta=-0.76, \mathrm{P}=0.009$ ) from baseline to follow-up. Significant improvements in patient-provider communication $(\mathrm{u} \Delta=0.46$, $\mathrm{P}=0.031)$ and work limitations from mental demands $(\mathrm{u} \Delta=-$ $8.89, \mathrm{P}=0.010)$ were also observed from baseline to followup. Relative to Usual Care, wCDSMP participants reported significantly larger improvements in fatigue, physical activity, soda/sugar beverage consumption, and work limitations from mental demands $(\mathrm{P}<0.05)$. The translation of Usual Care (content and format) has potential to improve health among employees with chronic conditions and increase uptake in workplace settings.

\section{INDEPENDENT ASSOCIATIONS OF APATHY AND DEPRESSIVE SYMPTOMS WITH PERCEIVED SOCIAL SUPPORT IN HEALTHY OLDER ADULTS \\ J. Lee ${ }^{1}$, R. Holtzer ${ }^{2}$, 1. Ferkauf Graduate School of \\ Psychology, 2. Albert Einstein College of Medicine}

Apathy syndrome is distinct from depression but apathy symptoms are present in both conditions. Moreover, apathy symptoms are also present in healthy older adults. Perceived social support is considered a protective factor against adverse health outcomes in the aging population. While numerous studies found depressive symptoms to be inversely associated with perceived social support, the relationship between apathy and perceived social support has not been reported. Hence, we investigated the associations of apathy and depressive symptoms with perceived social support in older adults. The sample included 295 community-dwelling healthy older adults (mean age $=78.29 \mathrm{yrs} ; \%$ female $=52.9 \%$ ). Apathy was evaluated with the Apathy Evaluation Scale, depression with the Geriatric Depression Scale- 30, and perceived social support with the Medical Outcomes Study- Social Support Survey. In order to adjust for the apathy symptoms found in depression, four apathy/withdrawal items were excluded, based on prior literature, from the Geriatric Depression Scale. Hierarchical linear regression analysis adjusting for age, gender, education, cognition, and medical comorbidities revealed that, when apathy and depressive symptoms were jointly modeled as predictors, both had significant negative associations with perceived social support. We provided the first evidence that worse apathy symptoms were associated with lower perceived social support in older adults independently of depressive symptoms. These findings suggest that apathy may be a novel risk factor for diminished social support and a plausible candidate for interventions designed to enhance it.

\section{LONELINESS TRAJECTORIES AMONG OLDER ADULT MARRIED COUPLES}

A. Ermer ${ }^{1}$, D. Segel-Karpas ${ }^{2}$, J. Benson 3 , 1. Montclair State

University, 2. University of Haifa, 3. University of Missouri

Loneliness is a significant social concern-being lonely is linked to depressive symptoms, mortality, and blood pressure (Cacioppo, Hughes, Waite, Hawkley, Thisted, 2006; Hawkley, Masi, Berry, \& Cacioppo, 2006; Luo, Hawkley, Waite, \& Cacioppo, 2012). How loneliness manifests in older adult couples is necessary to consider, especially given roughly 1 in 5 married older adults report social and emotional loneliness (de Jong Gierveld, Broese van Groenou, Hoogendoorn, \& Smit, 2009). Social contagion, the phenomenon where a behavior or feeling spreads to those close, is also a concern (see Christakis \& Fowler, 2013 for a review). Therefore, examining couples dyadically is beneficial to understand trajectories of loneliness. The present study used a personcentered approach to examine loneliness trajectories of older adult married couples. Three waves of the Health and Retirement Study, growth mixture modeling using Mplus version 7.4 (Muthen \& Muthen, 1998 - 2012) and ANOVAs were used. Three classes emerged including high loneliness, husband increasing, wife stable $(\mathrm{N}=69)$; low loneliness, husband decreasing, wife stable $(\mathrm{N}=998)$; and moderate loneliness, husband and wife increasing $(\mathrm{N}=322)$. Classes were distinguished at the first wave by husbands' activities of daily living, health conditions, divorce history, and religiosity; by wives' doctor usage, age, retirement, and family support; and by both husbands' and wives' depressive symptoms, self-rated health, life satisfaction, marital positivity and negativity. The present study gives insight into how loneliness in functions over time among older adult couples and has implications for practitioners who work with older adult couples.

\section{LONELY NIGHTS: THE RELATIONSHIP BETWEEN LONELINESS AND RESTLESS SLEEP IN OLDER AMERICANS}

S. Griffin, A. Baylor, B. Rybarczyk, J. Dzierzewski, Virginia Commonwealth University

Loneliness is a risk factor for morbidity and mortality, yet the mechanics behind this relationship remain unclear. One proposed mechanism is through sleep disturbance: there is evidence that lonely persons experience lower 